\title{
The Prognostic Impact of the Lymphocyte-to-Monocyte Ratio in Resected Pancreatic Head Adenocarcinoma
}

\author{
Shunsuke Onoe Atsuyuki Maeda Yuichi Takayama Yasuyuki Fukami \\ Takamasa Takahashi Masahito Uji Yuji Kaneoka \\ Department of Surgery, Ogaki Municipal Hospital, Ogaki, Japan
}

\section{Significance of the Study}

- This study aimed to investigate the prognostic value of the preoperative lymphocyte-to-monocyte ratio (LMR) in patients with resectable pancreatic head cancers. The LMR may carry important prognostic information in this condition.

\section{Keywords}

Lymphocyte-to-monocyte ratio - Pancreatic head adenocarcinoma. Systematic inflammation-based scores

\section{Abstract}

Objective: Although the prognostic significance of systematic inflammation-based scores, such as the neutrophil-tolymphocyte ratio (NLR), the platelet-to-lymphocyte ratio $(\mathrm{PLR})$, and the prognostic nutritional index (PNI), has been explored in pancreatic cancers, few reports have investigated the lymphocyte-to-monocyte ratio (LMR). We aimed to retrospectively investigate the prognostic value of the preoperative LMR in patients with resectable pancreatic head cancer (PHC). Methods: From 2005 to 2016, 165 patients underwent pancreatoduodenectomy for PHC. All samples of peripheral blood were collected within 2 weeks prior to surgery. The best cutoff values of the LMR for predicting sur-
}

vival were determined by using a minimum $p$ value approach (cut-off value: 2.8). The clinicopathological features of LMR $<2.8$ ( $n=25)$ and $\geq 2.8$ ( $n=140$ ) were compared. Results: Patients with $L M R \geq 2.8$ showed significantly lower NLR and PLR, and significantly higher PNI. Levels of CEA and CA19-9 were similar, and the pathological findings were comparable between the groups. The overall survival of patients with LMR $\geq 2.8$ (66.2\% at 1 year) was superior to that of patients with LMR $<2.8$ (36.1\% at 1 year, $p=0.015)$. Multivariate analysis identified LMR $<2.8$ (hazard ratio $1.72,95 \% \mathrm{Cl} 1.02-2.89$, $p=0.042$ ), lymphatic and venous invasion and positive surgical margin as independent prognostic factors. Conclusions: LMR may carry important prognostic information for patients with resectable PHC. Preoperative LMR may be considered for use in risk stratification for individual patients with $\mathrm{PHC}$.

(c) 2019 The Author(s)

Published by S. Karger AG, Basel

\begin{tabular}{ll}
\hline KARGER & $\begin{array}{l}\text { () } 2019 \text { @ } 2019 \text { The Author(s) } \\
\text { Published by S. Karger AG, Basel } \quad \text { Karger }\end{array}$ \\
$\begin{array}{l}\text { E-Mail karger@karger.com access } \\
\text { www.karger.com/mpp }\end{array}$ & $\begin{array}{l}\text { This is an Open Access article licensed under the Creative Commons } \\
\text { Attribution-NonCommercial-4.0 International License (CC BY-NC) } \\
\text { (http://www.karger.com/Services/OpenAccessLicense), applicable to } \\
\text { the online version of the article only. Usage and distribution for com- } \\
\text { mercial purposes requires written permission. }\end{array}$
\end{tabular}




\section{Introduction}

Recent studies have confirmed that cancer-related inflammation affects tumour malignancy, including tumour proliferation and survival, angiogenesis, metastasis, and response to treatment [1]. It was reported that the preoperative measurement of a variety of systematic inflammation-based prognostic scores, including the neutrophil-to-lymphocyte ratio (NLR), the platelet-to-lymphocyte ratio (PLR), the prognostic nutritional index (PNI), and the Glasgow prognostic score, can predict cancer-specific survival in several digestive cancers [2-8].

In recent years, the prognostic significance of these inflammation-based scores has also been explored in pancreatic cancers (PCs) [9-15]. Although surgical resection is the only available treatment option for cure or longterm survival for resectable and borderline resectable PCs, predicting the prognosis would be valuable for determining therapeutic strategies, that is, determining whether undergoing neoadjuvant treatment or upfront resection with adjuvant treatment would be more appropriate [16]. Among these systematic inflammation-based prognostic scores, monocyte levels have not been widely investigated as biomarkers in PC despite evidence implicating them in carcinogenesis. In particular, tumor-associated macrophages, which are derived from circulating monocyte populations, have been reported to be a key player in the tumor microenvironment, encouraging metastasis, and tumor progression [2,17]. Nonetheless, only a few reports to date have investigated the lymphocyteto-monocyte ratio (LMR) as a prognostic marker in patients with PC [18-20].

Here, we analysed the clinical features of pancreatic head carcinoma in a group of patients in a single institution who were treated by resection. The present study aimed to investigate the prognostic value of preoperative LMR in patients with resectable pancreatic head cancers compared with other inflammation-based prognostic markers, such as NLR, PLR, and PNI.

\section{Methods}

\section{Patient Characteristics}

We identified 165 consecutive patients who underwent pancreatoduodenectomy (PD) for pancreatic head adenocarcinoma from January 2005 to July 2016 in the Department of Surgery, Ogaki Municipal Hospital. This study was approved by the Human Research Review Committee of Ogaki Municipal Hospital. All peripheral blood samples were collected within 2 weeks prior to surgery. For patients with jaundice and/or cholangitis, either percutaneous transhepatic biliary drainage or endoscopic biliary drainage was routinely performed according to clinical indications. Every patient in this study underwent pancreatic resection after the preoperative serum bilirubin level was normalized $(\leq 2.0$ $\mathrm{mg} / \mathrm{dL}$ ).

None of the patients underwent diagnostic laparoscopy or peritoneal cytology. The operative procedures were divided into PD, pylorus-preserving $\mathrm{PD}$, and sub-stomach preserving $\mathrm{PD}$, according to the degree of direct invasion of the duodenum and the stomach. Reconstruction was performed using a modified Child's method; end-to-side pancreaticojejunostomy, biliojejunostomy, and gastro/duodenojejunostomy were performed using a jejunal limb in the order listed. Portal and/or superior mesenteric vein resection (PVR) was planned based on both preoperative multidetector-row computed tomography and the intraoperative findings of PV/SMV involvement, which indicated venous narrowing and/ or tumour abutment to the vein. The selection criteria for autologous vein graft reconstruction were the length of the PVR (longer than approximately $5 \mathrm{~cm}$ ) and the existence of venous collateral formation resulting from severe PV/SMV stenosis based on preoperative imaging [21]. PV/SMV invasion, defined as local cancer cell infiltration through the vessel wall, was evaluated microscopically for cases with PVR.

The surgical indications in each patient were discussed with a gastroenterologist; distant metastasis including para-aortic lymph node enlargement and apparent invasion of the celiac artery or SMA were considered contraindications for curative operation. However, cases with invasion to the plexus around the SMA were considered to be resectable.

For each of the 165 patients, operative specimens were cut at intervals of $0.5 \mathrm{~cm}$. Tumour size and pathological findings were assessed. Overall survival of patients was determined from the time of surgery to the time of death or the most recent follow-up.

\section{Definitions and Quantitative Determination of $L M R, N L R$,}

$P L R$, and PNI

Blood LMR was calculated as the ratio of the total count of lymphocytes divided by the total count of monocytes, NLR as the ratio of the total count of neutrophils divided by the total count of lymphocytes, and PLR as the ratio of the total count of platelets divided by the total count of lymphocytes. PNI was calculated as albumin $(\mathrm{g} / \mathrm{L})+0.005 \times$ lymphocyte count $\times 10^{9} / \mathrm{L}$. Blood samples were obtained from patients within 2 weeks prior to surgery. For patients with inflammation (such as cholangitis), which affects blood counts, the normalized blood counts after biliary drainage were evaluated.

Concerning the impact of these systematic inflammationbased scores on survival, the "minimum $p$ value" approach, which was performed using the log-rank test for the overall survival, was used to determine the best cutoff values of each score for predicting survival for the entire cohort.

\section{Statistical Analysis}

Measurement values for continuous variables are expressed as the median (range). The characteristics were compared using the Kruskal-Wallis test for continuous variables and the $\chi^{2}$ test for categorical variables. In addition, Fisher's exact test was applied where appropriate. Postoperative survival was calculated using the Kaplan-Meier method. Differences in survival curves were compared using the log-rank test. Multivariate survival analyses were performed using the Cox proportion hazards regression model. 
Table 1. Clinical characteristics of the 165 patients in this study

\begin{tabular}{lc}
\hline & $n=165$ \\
\hline Age, years & $72(45-83)$ \\
Gender & \\
Men & $89(54)$ \\
Women & $76(46)$ \\
BMI, kg/m ${ }^{2}$ & $20.8(16.4-30.2)$ \\
Surgical procedure & \\
PD & $17(10)$ \\
PpPD & $86(52)$ \\
SSpPD & $62(38)$ \\
PVR & \\
None & $61(37)$ \\
End-to-end anastomosis & $79(48)$ \\
Graft construction & $25(15)$ \\
Operative time, min & $299(198-664)$ \\
Blood loss, mL & $410(100-4,900)$ \\
Blood transfusion & \\
Absent & $147(89)$ \\
Present & $18(11)$ \\
Adjuvant chemotherapy & \\
Neoadjuvant therapy & $8(5)$ \\
Postoperative adjuvant therapy & $66(40)$ \\
Histological type & \\
Well differentiated & $12(7)$ \\
Moderately differentiated & $129(78)$ \\
Poorly differentiated & $16(5)$ \\
Other & \\
\hline
\end{tabular}

Expressed as $n(\%)$ or median (range).

$\mathrm{PD}$, pancreatoduodenectomy; PpPD, pylorus-preserving PD; SSpPD, sub-stomach preserving PD; PVR, portal vein resection; $\mathrm{SMV}$, superior mesenteric vein; BMI, body mass index.

For the multivariate model, backward variable selection was used to identify independent prognostic factors. The assumptions of proportional hazards were checked by the time-dependent Cox regression model. The results were considered statistically significant if the $p$ value was $<0.05$. All calculations were performed using the IBM SPSS Statistics 23 software package (IBM Japan Inc., Tokyo, Japan).

\section{Results}

Clinical characteristics of the entire cohort are shown in Table 1. At the time of operative exploration, the median age of all patients was 72 years, and the patient cohort included 89 men and 76 women. PVR was performed in $104(63 \%)$ patients. Neoadjuvant and postoperative adjuvant chemotherapy was administered in 8 and 66 patients (5 and 40\%) respectively. None underwent adju-

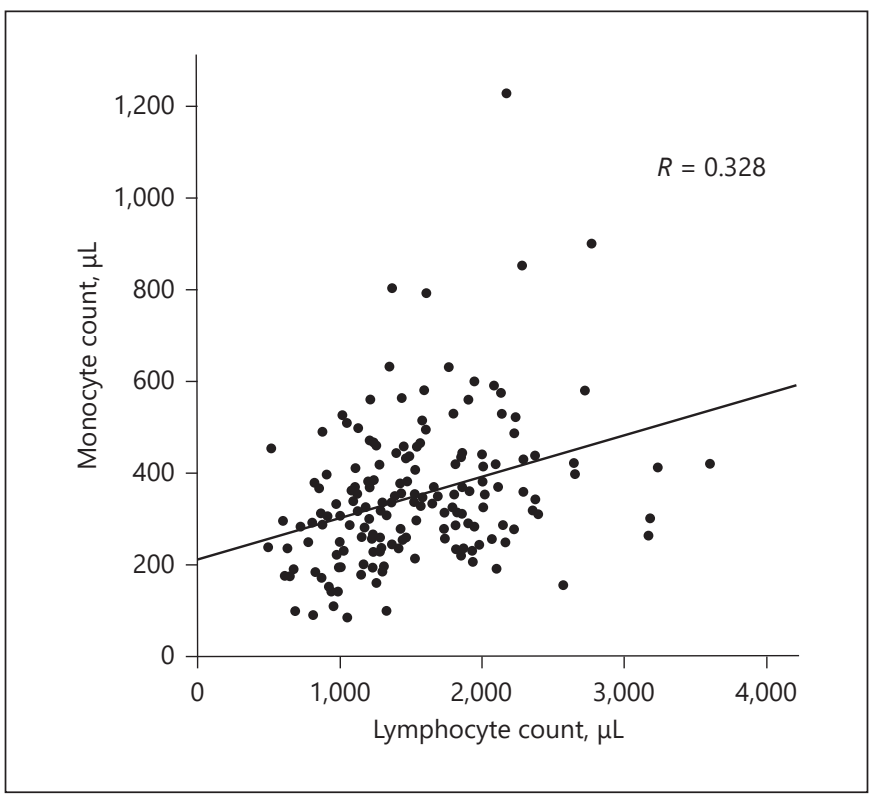

Fig. 1. Correlation between lymphocyte and monocyte counts.

vant radiotherapy. The median follow-up period was 1,788 days, and 106 (64\%) patients died in the follow-up period.

\section{Cut-Off Values of LMR, NLR, PLR, and PNI}

We first examined the correlation between LMR levels and overall survival in the cohort of 165 cases. There was weak positive correlation between lymphocyte and monocyte counts (Fig. 1). The best cutoff values of LMR for predicting survival were determined by using a minimum $p$ value approach for the entire cohort. When sliding the cutoff value of LMR from 2.0 to 5.0, the minimum $p$ values were obtained at 2.8 (Fig. 2a), which was comparable with the cutoff value found in the previous series $[2,18-$ 20]. In the same way, optimal cutoff values of NLR, PLR, and PNI were assessed (Fig. 2b-d).

\section{Comparison between $L M R<2.8$ and $\geq 2.8$}

Table 2 shows the comparison of the clinicopathological features of LMR $<2.8(n=25)$ and $\geq 2.8(n=140)$. The age distributions were similar between the 2 groups, whereas the proportion of men was larger in LMR $<2.8$. Betweengroup differences were observed in systematic inflammation markers such as NLR, PLR, and PNI. Patients with a greater LMR showed a decreased likelihood of NLR and PLR and an increased likelihood of PNI. In contrast, the CEA and CA19-9 levels were similar. The pathological findings were comparable between the groups. 


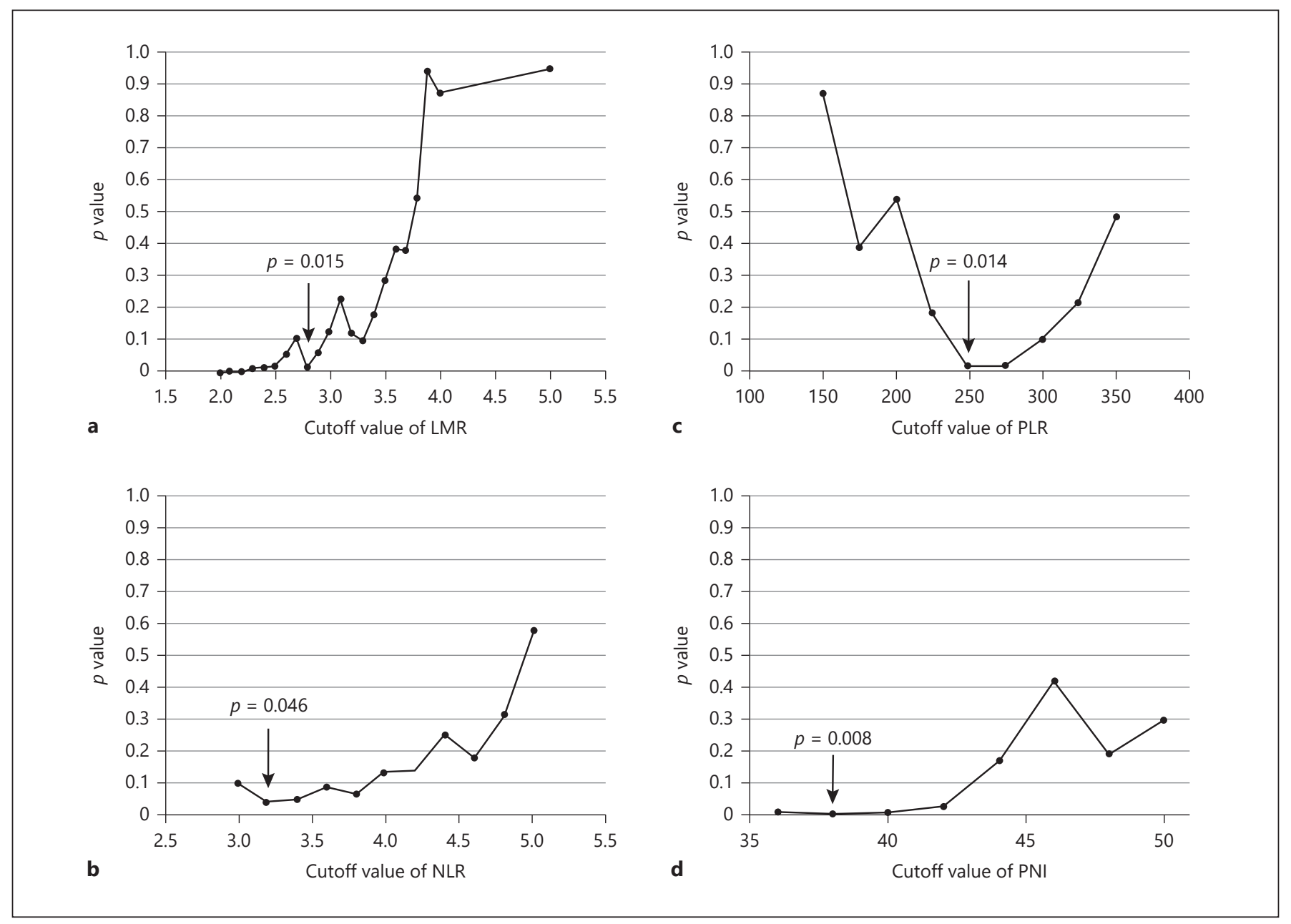

Fig. 2. a Optimal cutoff value of LMR for predicting overall survival. When testing LMR cutoff values from 2.0 to 5.0, the minimum $p$ value was obtained at an LMR cutoff of 2.8 (arrow). b-d In the same way, optimal cut-off values of NLR, PLR, and PNI were assessed (arrows). LMR, lymphocyte-to-monocyte ratio; NLR, neutrophil-tolymphocyte ratio; PLR, platelet-to-lymphocyte ratio; PNI, prognostic nutritional index.

The overall survival of patients with $\mathrm{LMR} \geq 2.8$ was superior to that of patients with LMR $<2.8$ ( $p=0.015$; Fig. 3a), in which the proportional hazard assumption was fulfilled $(p=0.946)$. The 1 -year survival rate and median survival time were $66.2 \%$ and 565 days in patients with LMR $\geq 2.8$ and $36.1 \%$ and 267 days in patients with LMR $<2.8$ respectively.

Three preoperative laboratory variables, one operative procedure and 12 postoperative pathological variables were analysed as potential prognostic factors in the 165-patient cohort (Table 3). The univariate analysis showed that 10 of the 16 variables were potential predictors of survival. The multivariate analysis identified 4 of these, that is, $\mathrm{LMR}<2.8$ (preoperative variable), lymphat- ic and venous invasion (postoperative variables), and positive surgical margin (postoperative variables), as independent prognostic factors.

\section{Assessment of NLR, PLR, and PNI}

Because LMR was strongly correlated with NLR, PLR, and PNI (online suppl. Fig. 1; for all online suppl. material, see www.karger.com/doi/10.1159/000501017), the data were analysed separately.

The survival rates of NLR $<3.2$, PLR $<250$, and PNI $\geq 38$ were significantly better than those of NLR $\geq 3.2$, PLR $<250$, and PNI $<38$, respectively (Fig. 3b-d). Multivariate analysis identified all 3 variables as independent prognostic factors (online suppl. Tables 1-3). 
Table 2. Clinicopathological features of patients according to the LMR

\begin{tabular}{|c|c|c|c|}
\hline & LMR <2.8 $(n=25)$ & $\mathrm{LMR} \geq 2.8(n=140)$ & $p$ value \\
\hline Age, years & $68(52-81)$ & $72(40-85)$ & 0.174 \\
\hline Gender & & & 0.049 \\
\hline Men & $18(72)$ & $71(51)$ & \\
\hline Women & $7(28)$ & $69(49)$ & \\
\hline Size, $\mathrm{cm}$ & & & 0.176 \\
\hline$<2.0$ & $1(4)$ & $18(13)$ & \\
\hline$\geq 2.0$ & $24(96)$ & $122(87)$ & \\
\hline Neutrophil count $(/ \mu \mathrm{L})$ & $4,343(2,071-9,464)$ & $3,348(911-8,820)$ & 0.002 \\
\hline Platelet count $\left(\times 10^{4} / \mu \mathrm{L}\right)$ & $24.3(12.3-39.8)$ & $24.7(7.0-52.8)$ & 0.989 \\
\hline Lymphocyte count $(/ \mu \mathrm{L})$ & $1,103(499-2,275)$ & $1,524(615-3,595)$ & $<0.001$ \\
\hline Monocyte count $(/ \mu \mathrm{L})$ & $470(234-1,227)$ & $312(85-900)$ & $<0.001$ \\
\hline NLR & $4.0(1.5-8.9)$ & $2.2(0.5-6.9)$ & $<0.001$ \\
\hline PLR & $249(102-608)$ & $160(37-551)$ & 0.001 \\
\hline PNI & $44.2(36.4-56.4)$ & $47.8(29.9-66.0)$ & 0.023 \\
\hline CEA, ng/dL & $3.2(1.0-77.0)$ & $3.2(1.0-74.0)$ & 0.900 \\
\hline CA19-9, U/mL & $239.0(2.0-3,768.0)$ & $126.1(2.0-15,760.0)$ & 0.243 \\
\hline \multicolumn{4}{|l|}{ Pathological findings (present) } \\
\hline Lymphatic invasion & $23(92)$ & $128(91)$ & 0.642 \\
\hline Venous invasion & $18(72)$ & $78(58)$ & 0.128 \\
\hline Perineural invasion & $25(100)$ & $126(90)$ & 0.090 \\
\hline Anterior serosal infiltration & $14(56)$ & $66(47)$ & 0.414 \\
\hline Retroperitoneal invasion & $19(76)$ & $94(67)$ & 0.380 \\
\hline Bile duct invasion & $17(68)$ & $88(63)$ & 0.622 \\
\hline Duodenal invasion & $13(52)$ & $72(51)$ & 0.958 \\
\hline Plexus invasion & $9(36)$ & $52(37)$ & 0.913 \\
\hline $\mathrm{PV} / \mathrm{SMV}$ invasion & $11(44)$ & $48(34)$ & 0.351 \\
\hline Lymph node metastasis & $17(68)$ & $94(67)$ & 0.933 \\
\hline Surgical margin (positive) & $9(36)$ & $26(19)$ & 0.050 \\
\hline
\end{tabular}

Expressed as $n(\%)$ or median (range).

NLR, neutrophil-to-lymphocyte ratio; PLR, platelet-to-lymphocyte ratio; PNI, prognostic nutritional index; PV, portal vein; SMV, superior mesenteric vein; LMR, lymphocyte-to-monocyte ratio.

\section{Discussion}

Our findings demonstrate that preoperative LMR is an independent predictor of overall survival for patients who have undergone PD for pancreatic head adenocarcinoma, as well as other inflammation-based prognostic markers, such as NLR, PLR, and PNI. To the best of our knowledge, this is the first report to correlate overall survival after operative resection of pancreatic "head" adenocarcinoma with preoperative LMR. We excluded PCs requiring distal pancreatectomy when we investigated prognostic markers because carcinomas of the body and tail are particularly associated with poor prognosis, even more so than carcinoma of the pancreatic head, due to a lack of symptoms such as obstructive jaundice and consequent difficulty in early detection [22]. In addition, resectable adjacent organs and vessels in cancers of the pancreatic head are distinctively different from those in cancers of the body and tail $[16,22,23]$. Therefore, we focused on only pancreatic "head" adenocarcinoma in the present series, although 2 studies previously reported that LMR was a prognosticator after surgical resection in PCs including those of the body and tail $[18,19]$. Recently, the preoperative NLR, PLR, and PNI have been actively studied in PCs [9-15]. However, the multivariate analysis in this cohort revealed that the LMR could be a predictor of survival (Table 3), as well as the NLR, the PLR, and the PNI (online suppl. Table 1-3). Notably, these scores, which are routinely measured by laboratory tests, are simple and inexpensive. Further, our data showed that preoperative CEA and CA19-9 were not prognosticators in either the univariate or multivariate analysis. Besides, the optimal 
Table 3. Univariate and multivariate analyses in 165 patients with resected pancreatic head carcinoma

\begin{tabular}{|c|c|c|c|c|c|c|}
\hline \multirow[t]{2}{*}{ Variable } & \multirow[t]{2}{*}{ Patients } & \multicolumn{2}{|c|}{ Survival, \% } & \multirow{2}{*}{$\begin{array}{l}p \text { (Log-rank } \\
\text { test) }\end{array}$} & \multicolumn{2}{|l|}{ Multivariate } \\
\hline & & 1-year & 2-year & & $\begin{array}{l}\text { hazard ratio } \\
(95 \% \mathrm{CI})\end{array}$ & $p$ value \\
\hline LMR & & & & 0.015 & & 0.042 \\
\hline$<2.8$ & 25 & 36.1 & 18.6 & & $1.72(1.02-2.89)$ & \\
\hline$\geq 2.8$ & 140 & 66.2 & 40.5 & & 1.00 & \\
\hline CEA, ng/dL & & & & 0.120 & & \\
\hline$\leq 5.0$ & 121 & 58.6 & 34.3 & & & \\
\hline$>5.0$ & 40 & 71.1 & 43.3 & & & \\
\hline CA19-9, U/mL & & & & 0.057 & & \\
\hline$<37$ & 40 & 74.7 & 46.1 & & & \\
\hline$\geq 37$ & 120 & 56.7 & 32.7 & & & \\
\hline PV resection & & & & $<0.001$ & & \\
\hline None & 61 & 80.4 & 56.7 & & & \\
\hline Resected & 104 & 51.3 & 26.0 & & & \\
\hline Size, $\mathrm{cm}$ & & & & 0.069 & & \\
\hline$\leq 2.0$ & 19 & 94.4 & 63.8 & & & \\
\hline$>2.0$ & 146 & 57.6 & 33.9 & & & \\
\hline Lymphatic invasion & & & & 0.001 & & 0.030 \\
\hline Absent & 14 & 92.3 & 92.3 & & 1.00 & \\
\hline Present & 151 & 59.1 & 31.9 & & $2.81(1.17-7.12)$ & \\
\hline Venous invasion & & & & $<0.001$ & & 0.006 \\
\hline Absent & 69 & 76.1 & 54.6 & & 1.00 & \\
\hline Present & 96 & 51.0 & 23.3 & & $1.87(1.19-2.93)$ & \\
\hline Perineural invasion & & & & 0.001 & & \\
\hline Absent & 14 & 100.0 & 90.0 & & & \\
\hline Present & 151 & 58.4 & 32.9 & & & \\
\hline Anterior serosal infiltration & & & & 0.003 & & \\
\hline Absent & 85 & 70.7 & 45.1 & & & \\
\hline Present & 80 & 52.0 & 28.3 & & & \\
\hline Retroperitoneal invasion & & & & $<0.001$ & & \\
\hline Absent & 52 & 78.4 & 61.3 & & & \\
\hline Present & 113 & 54.6 & 26.5 & & & \\
\hline Bile duct invasion & & & & 0.135 & & \\
\hline Absent & 60 & 68.6 & 44.7 & & & \\
\hline Present & 105 & 58.2 & 33.2 & & & \\
\hline Duodenal invasion & & & & 0.428 & & \\
\hline Absent & 80 & 63.5 & 42.6 & & & \\
\hline Present & 85 & 60.4 & 32.2 & & & \\
\hline Plexus invasion & & & & $<0.001$ & & \\
\hline Absent & 104 & 70.4 & 47.5 & & & \\
\hline Present & 61 & 48.4 & 20.5 & & & \\
\hline $\mathrm{PV} / \mathrm{SMV}$ invasion & & & & $<0.001$ & & \\
\hline Absent & 106 & 72.2 & 45.6 & & & \\
\hline Present & 59 & 44.3 & 23.2 & & & \\
\hline Lymph node metastasis & & & & 0.002 & & \\
\hline Absent & 54 & 72.5 & 53.9 & & & \\
\hline Present & 111 & 56.8 & 29.4 & & & \\
\hline Surgical margin & & & & $<0.001$ & & $<0.001$ \\
\hline Negative & 130 & 73.5 & 45.5 & & 1.00 & \\
\hline Positive & 35 & 19.5 & 7.8 & & $2.88(1.81-4.59)$ & \\
\hline
\end{tabular}

Expressed as $n$ (\%) or median (range).

LMR, lymphocyte-to-monocyte ratio; NLR, neutrophil-to-lymphocyte ratio; PLR, platelet-to-lymphocyte ratio; PNI, prognostic nutritional index; PV, portal vein; SMV, superior mesenteric vein. 


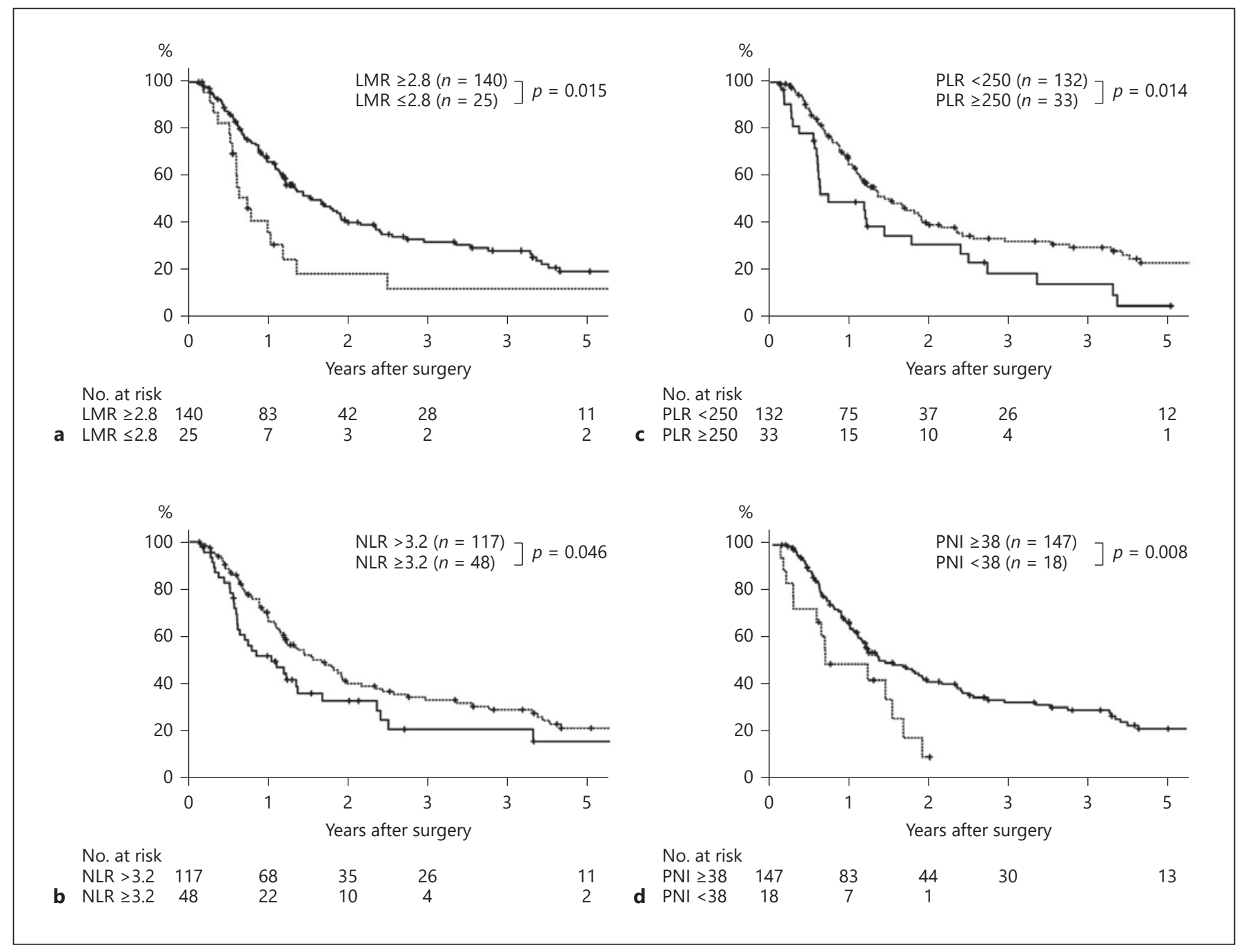

Fig. 3. a Cumulative overall survival rates of patients in PHC with LMR $\geq 2.8$ and that of patients with LMR $<2.8$. Log-rank test. b Cumulative overall survival rates of patients in PHC with NLR $\geq 3.2$ and that of patients with NLR $<3$.2. Log-rank test. c Cumulative overall survival rates of patients in PHC with PLR $\geq 250$ and

cut-off value of neutrophil, platelet, lymphocyte, and monocyte counts were investigated using the minimum $p$ value approach method; however, no value could be considered statistically significant. Although several histologic parameters of resected PC specimens have been shown to represent significant prognostic variables, these parameters are invariably amenable to assessment only after surgery. Consequently, the LMR could be one of the most significant inflammation-based prognostic scores in resectable pancreatic head carcinoma.

The LMR in Pancreatic Head Adenocarcinoma that of patients with PLR $<250$. Log-rank test. d Cumulative overall survival rates of patients in PHC with PNI $\geq 38$ and that of patients with PNI <38. Log-rank test. LMR, lymphocyte-to-monocyte ratio; NLR, neutrophil-to-lymphocyte ratio; PLR, platelet-tolymphocyte ratio; PNI, prognostic nutritional index.

This study revealed that there was no significant association between LMR and pathological findings, although a low LMR can be considered one of the reliable prognostic biomarker.

An exact preoperative evaluation of tumors based on radiographic findings is difficult or even impossible [16], as radiographic findings do not often coincide with pathological findings; thus, the LMR could be useful when considering the malignant potential of pancreatic head adenocarcinomas. A recent large study of colorectal cancer reported that there was some association between the 
LMR and pathological findings [2]; that study demonstrated that a low LMR was associated with more advanced colorectal tumors. The differences between pancreatic and colorectal cancers are thus unclear in these findings. However, they could suggest that pancreatic tumors are more susceptible to mediators of inflammation than colorectal cancers. Moreover, in the present study, potential prognostic markers such as CEA and CA19-9 were comparable between low and high LMR [24]; the LMR may be a more sensitive prognostic marker than CEA and CA19-9. Notably, the present series showed a positive correlation with PNI, and a negative correlation with both NLR and PLR. Thus, our study supports the prognostic predictive value of LMR in resectable pancreatic head adenocarcinomas.

We defined an optimal cutoff point for the LMR of 2.8 in pancreatic head adenocarcinoma. To date, few studies have investigated the association between LMR and survival in resectable PCs, although NLR and PLR were frequently evaluated. Two studies on groups of 144 and 442 patients with curatively resected PCs showed that LMR $<2.86$ and $<3$ were independent prognostic factors respectively $[18,19]$. A similar observation was reported in another study of 474 patients who underwent either curative resection or palliative chemotherapy, where LMR $>2.8$ indicated a reduced risk of death [20]. In a large colorectal cancer series, a cutoff point of 2.38 for the LMR was found to have the highest log-rank statistic of any cutoff point [2]. Therefore, our cutoff value of 2.8, which was determined by the minimum $p$ value approach, is a valid result. Of note, the minimum $p$ value approach has been proposed as a means of reducing the risk of missing a significant association [25]. However, this approach may give false positive associations. Because there is no agreement on the best way of determining a cutoff value, the potential validity and use of such an approach should be further tested.
Our study has some limitations. First, this was a retrospective study performed over a long period of time, which included a small population with a low rate of adjuvant chemotherapy. Our model did not identify several potential predictors of survival, that is, lymph node metastases, invasion to the portal vein, and so on, as predictors for mortality. This lack of identifying certain predictors is likely not because these factors are not predictors but rather because the present sample size was too small. Nevertheless, the importance of LMR was shown. Second, LMR could not provide a specific strategy for treatment of PC. Because the benefit of evaluating inflammation-based prognostic markers remains unclear, further prospective studies and/or multi-institutional studies are needed to clarify their clinical value. Third, CRP was not routinely examined in our patient cohort, although previous studies have confirmed that the Glasgow prognostic score and the prognostic index based on CRP were useful and important predictors of overall survival in PC [10, 11]. To compare these indexes with LMR, additional cohort studies are needed.

\section{Conclusion}

LMR may provide important prognostic information for patients with resectable pancreatic head adenocarcino$\mathrm{ma}$, as well as other inflammation-based prognostic markers. Our study confirmed that preoperative LMR can be used to enable optimal risk stratification of individual patients prior to treatment and to predict post-curative prognosis of patients with pancreatic head adenocarcinoma.

\section{Disclosure Statement}

The authors indicate no potential conflict of interest.

\section{References}

1 Mantovani A, Allavena P, Sica A, Balkwill F. Cancer-related inflammation. Nature. 2008 Jul;454(7203):436-44.

2 Chan JC, Chan DL, Diakos CI, Engel A, Pavlakis N, Gill A, et al. The Lymphocyte-toMonocyte Ratio is a Superior Predictor of Overall Survival in Comparison to Established Biomarkers of Resectable Colorectal Cancer. Ann Surg. 2017 Mar;265(3):539-46.
3 Okuno M, Ebata T, Yokoyama Y, Igami T, Sugawara G, Mizuno T, et al. Evaluation of inflammation-based prognostic scores in patients undergoing hepatobiliary resection for perihilar cholangiocarcinoma. J Gastroenterol. 2016 Feb;51(2):153-61.

4 Okuno M, Ebata T, Yokoyama Y, Igami T, Sugawara G, Mizuno T, et al. Appraisal of inflammation-based prognostic scores in patients with unresectable perihilar cholangiocarcinoma. J Hepatobiliary Pancreat Sci. 2016 Oct;23(10):636-42.
5 Templeton AJ, McNamara MG, Šeruga B, Vera-Badillo FE, Aneja P, Ocaña A, et al. Prognostic role of neutrophil-to-lymphocyte ratio in solid tumors: a systematic review and metaanalysis. J Natl Cancer Inst. 2014 May; 106(6):dju124.

6 Templeton AJ, Ace O, McNamara MG, AlMubarak M, Vera-Badillo FE, Hermanns T, et al. Prognostic role of platelet to lymphocyte ratio in solid tumors: a systematic review and meta-analysis. Cancer Epidemiol Biomarkers Prev. 2014 Jul;23(7):1204-12. 
7 Kinoshita A, Onoda H, Imai N, Iwaku A, Oishi M, Fushiya N, et al. Comparison of the prognostic value of inflammation-based prognostic scores in patients with hepatocellular carcinoma. Br J Cancer. 2012 Sep;107(6): 988-93.

8 Dutta S, Crumley AB, Fullarton GM, Horgan PG, McMillan DC. Comparison of the prognostic value of tumour- and patient-related factors in patients undergoing potentially curative resection of oesophageal cancer. World J Surg. 2011 Aug;35(8):1861-6.

9 Gemenetzis G, Bagante F, Griffin JF, Rezaee N, Javed AA, Manos LL, et al. Neutrophil-tolymphocyte Ratio is a Predictive Marker for Invasive Malignancy in Intraductal Papillary Mucinous Neoplasms of the Pancreas. Ann Surg. 2017 Aug;266(2):339-45.

10 Yamada S, Fujii T, Yabusaki N, Murotani K, Iwata N, Kanda M, et al. Clinical Implication of Inflammation-Based Prognostic Score in Pancreatic Cancer: Glasgow Prognostic Score Is the Most Reliable Parameter. Medicine (Baltimore). 2016 May;95(18):e3582.

11 Watanabe J, Otani S, Sakamoto T, Arai Y, Hanaki T, Amisaki M, et al. Prognostic indicators based on inflammatory and nutritional factors after pancreaticoduodenectomy for pancreatic cancer. Surg Today. 2016 Nov; 46(11):1258-67.

12 Shirai Y, Shiba H, Sakamoto T, Horiuchi T, Haruki K, Fujiwara Y, et al. Preoperative platelet to lymphocyte ratio predicts outcome of patients with pancreatic ductal adenocarcinoma after pancreatic resection. Surgery. 2015 Aug;158(2):360-5.
13 Geng Y, Qi Q, Sun M, Chen H, Wang P, Chen Z. Prognostic nutritional index predicts survival and correlates with systemic inflammatory response in advanced pancreatic cancer. Eur J Surg Oncol. 2015 Nov;41(11):1508-14.

14 Kanda M, Fujii T, Kodera Y, Nagai S, Takeda S, Nakao A. Nutritional predictors of postoperative outcome in pancreatic cancer. $\mathrm{Br} \mathrm{J}$ Surg. 2011 Feb;98(2):268-74.

15 Smith RA, Bosonnet L, Raraty M, Sutton R, Neoptolemos JP, Campbell F, et al. Preoperative platelet-lymphocyte ratio is an independent significant prognostic marker in resected pancreatic ductal adenocarcinoma. Am J Surg. 2009 Apr;197(4):466-72.

16 Onoe S, Kaneoka Y, Maeda A, Takayama Y, Fukami Y. Preoperative radiographic vascular involvement score predicts the prognosis of resected pancreatic head adenocarcinoma. Langenbecks Arch Surg. 2017 May;402(3): 439-46.

17 Galdiero MR, Bonavita E, Barajon I, Garlanda C, Mantovani A, Jaillon S. Tumor associated macrophages and neutrophils in cancer. Immunobiology. 2013 Nov;218(11):1402-10.

18 Sierzega M, Lenart M, Rutkowska M, Surman M, Mytar B, Matyja A, et al. Preoperative Neutrophil-Lymphocyte and LymphocyteMonocyte Ratios Reflect Immune Cell Population Rearrangement in Resectable Pancreatic Cancer. Ann Surg Oncol. 2017 Mar;24(3): 808-15.
19 Li GJ, Xu HW, Ji JJ, Yang F, Gao BQ. Prognostic value of preoperative lymphocyte-tomonocyte ratio in pancreatic adenocarcinoma. Onco Targets Ther. 2016 Mar;9:1085-92.

20 Stotz M, Szkandera J, Stojakovic T, Seidel J, Samonigg H, Kornprat P, et al. The lymphocyte to monocyte ratio in peripheral blood represents a novel prognostic marker in patients with pancreatic cancer. Clin Chem Lab Med. 2015 Feb;53(3):499-506.

21 Kaneoka Y, Yamaguchi A, Isogai M. Portal or superior mesenteric vein resection for pancreatic head adenocarcinoma: prognostic value of the length of venous resection. Surgery. 2009 Apr; 145(4):417-25.

22 Kanda M, Fujii T, Sahin TT, Kanzaki A, Nagai $\mathrm{S}$, Yamada $\mathrm{S}$, et al. Invasion of the splenic artery is a crucial prognostic factor in carcinoma of the body and tail of the pancreas. Ann Surg. 2010 Mar;251(3):483-7.

23 Fukami Y, Kaneoka Y, Maeda A, Takayama Y, Onoe S. Prognostic impact of splenic artery invasion for pancreatic cancer of the body and tail. Int J Surg. 2016 Nov;35:64-8.

24 Kau SY, Shyr YM, Su CH, Wu CW, Lui WY. Diagnostic and prognostic values of CA 19-9 and CEA in periampullary cancers. J Am Coll Surg. 1999 Apr;188(4):415-20.

25 Yamamoto Y, Ikoma H, Morimura R, Konishi H, Murayama Y, Komatsu S, et al. Optimal duration of the early and late recurrence of hepatocellular carcinoma after hepatectomy. World J Gastroenterol. 2015 Jan;21(4):120715. 